\title{
Small scale lateral superlattices in two-dimensional electron gases prepared by diblock copolymer masks
}

\author{
S. Hugger and T. Heinze* \\ Solid State Physics Laboratory (IPkM), Heinrich-Heine Universität Düsseldorf, Universitätsstr. 1, 40225 Düsseldorf, Germany \\ T. Thurn-Albrecht \\ Institut für Physik, Martin-Luther-Universität Halle-Wittenberg, 06099 Halle, Germany
}

(Dated: October 24, 2018)

\begin{abstract}
A poly(styrene-block-methylmethacrylate) diblock copolymer in the hexagonal cylindrical phase has been used as a mask for preparing a periodic gate on top of a $\mathrm{GaAs} / \mathrm{Al}_{\mathrm{x}} \mathrm{Ga}_{1-\mathrm{x}} \mathrm{As}$-heterostructure. A superlattice period of $43 \mathrm{~nm}$ could be imposed onto the two-dimensional electron gas. Transport measurements show a characteristic positive magnetoresistance around zero magnetic field which we interpret as a signature of electron motion guided by the superlattice potential.
\end{abstract}

PACS numbers: 73.23.-b, 73.63.-b,62.23.St

Keywords: diblock copolymers, superlattices, magnetotransport

Lateral superlattices (LSLs) defined in twodimensional electron gases (2DEGs) have revealed a wide range of fascinating semiclassical and quantum transport phenomena, like commensurability resonances, [1, 2, 3] Aharonov - Bohm type oscillations [4, [5], the demonstration of the quasi-particle character of composite fermions, 6 6 or the fractal Hofstadter butterfly energy spectrum. [7, 8, 9] Recent theoretical suggestions regarding the implementation of spin qubits are based on small-period, high quality LSLs. [10] For studying LSLs in the quantum regime, a lattice constant comparable to the Fermi wavelength of the electrons, namely in the range of $30 \mathrm{~nm}$ to $50 \mathrm{~nm}$ in typical 2DEGs, is desirable. However, established lithographic techniques have been limited to lattice constants above $\approx 80 \mathrm{~nm}$. [8] Patterning techniques based on self-organization have the potential to reduce the LSL period significantly, and diblock copolymers (DBCs) are promising template materials. They offer three potentially useful phases (spherical, lamellar and hexagonal cylindrical) with lattice constants down to $20 \mathrm{~nm}$ and have already been incorporated in various processing schemes. 11] Moreover, LSLs have already been prepared by transferring the pattern from the spherical phase of a DBC into a 2DEG. 12]

Here, we report the preparation of a LSL with a period of $\mathrm{a}=43 \mathrm{~nm}$ in a 2DEG by using the hexagonal cylindrical phase of poly(styrene-block-methylmethacrylate) (PS-b-PMMA) to pattern a gate electrode. It is thereby demonstrated that all the process steps involved are compatible with the established GaAs processing technology. As compared to the technique of Melinte et al., [12] our method is complementary since the copolymer mask has a strictly two-dimensional character with columns oriented perpendicular to the sample surface and allows various processing options. Also, in our system the disorder does not seem to dominate over the induced periodic modulation in small magnetic fields.
The pattern transfer into the 2DEG is demonstrated by transport measurements, which show a characteristic positive magnetoresistance around $B=0$. We support this interpretation by numerical simulations within the Kubo formalism.

The experiments have been performed on a commercially available heterostructure 13] with the heterointerface $65 \mathrm{~nm}$ below the surface. The density and the mobility of the 2DEG at liquid helium temperatures are $2.3 \times 10^{15} \mathrm{~m}^{-2}$ and $28 \mathrm{~m}^{2} \mathrm{~V}^{-1} \mathrm{~s}^{-1}$, respectively. Hall bars of $10 \mu \mathrm{m}$ width and a separation of $13 \mu \mathrm{m}$ between the voltage probes were prepared by optical lithography and wet chemical etching. A PS-b-PMMA DBC with a molecular weight of $67 \mathrm{~kg} / \mathrm{mol}$ of the weight ratio PS : PMMA $=46: 21$ was dissolved in toluene. DBC films of $\approx 500 \mathrm{~nm}$ thickness were prepared on the preprocessed heterostructure by spin casting. In the melt, the DBC adopts a cylindrical morphology with a local hexagonal arrangement of PMMA cylinders embedded in a PS matrix and lattice constant of $43 \pm 2 \mathrm{~nm}$, in which the orientation of the PMMA cylinders is isotropic. Usage of the DBC film as a lithographic mask requires the cylinders to be oriented perpendicular to the substrate. This was obtained by annealing the films under an applied electric field of $40 \mathrm{~V} / \mu \mathrm{m}$ at $185^{\circ} \mathrm{C}$ for $16 \mathrm{~h}$ in nitrogen atmosphere. [14, 15] For this purpose, an aluminum layer on top of a kapton foil coated with a crosslinked Polydimethylsiloxane (PDMS) layer of $\approx 20 \mu \mathrm{m}$ thickness on the side towards the DBC film acted as the counter electrode, 14] see Fig. 1(a). While this process does not alter the thickness of the DBC film, it imposes a roughness of $\approx 20 \mathrm{~nm}$. The oriented films were exposed to an electron beam with an energy of $20 \mathrm{keV}$ and a dose of $50 \mu \mathrm{C} / \mathrm{cm}^{2}$. The effect of the electrons is to chop the PMMA while at the same time crosslinking the PS. Afterwards, the film was developed in acetic acid for two hours, during which the PMMA chains were removed, leaving behind a PS film with 

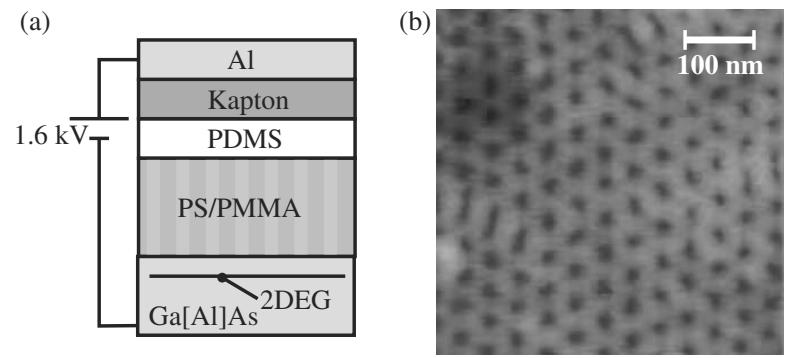

FIG. 1: (color online) (a) Cross-sectional scheme of the setup used for the orientation of the copolymer film. (b): Topography scan, taken with an atomic force microscope, of the oriented DBC film on top of the $\mathrm{Ga}[\mathrm{Al}] \mathrm{As}$ heterostructure, after removal of the PMMA cylinders.

holes in a hexagonal lattice geometry, see Fig. 1(b). The PS matrix shows characteristic deviations from a perfect hexagonal lattice. Namely, the orientation of the lattice vectors varies slowly and occasionally, grain boundaries are observed. Furthermore, about $1 \%$ of the holes are merged with a neighbor.

The gate electrode was electrodeposited from a saturated $\mathrm{CuSO}_{4}$ solution in water with $25 \%$ of methanole added. 15] The methanole reduces the capillary forces such that the solution penetrates into the cylindrical holes. A droplet of this solution was placed on top of the PS film and a voltage of $+3 \mathrm{~V}$ was applied to the droplet with respect to the grounded 2DEG, which was accessed via a standard Ohmic contact. The precipitation was stopped as soon as the cylindrical holes were filled and a homogeneous $\mathrm{Cu}$ electrode covered the PS film. The fact that $\mathrm{Cu}$ could be deposited this way indicates that the holes in the PS film extend all the way down to the GaAs surface.

Transport measurements were performed in a ${ }^{4} \mathrm{He}$ gas flow cryostat at a temperature of $2 \mathrm{~K}$ using standard lockin techniques with an excitation current of $100 \mathrm{nA}$ at a frequency of $13.6 \mathrm{~Hz}$. In Fig. 2, measurements of the longitudinal magnetoresistance are shown for different gate voltages. The average electron density as determined from Hall measurements was $1.8 \times 10^{15} \mathrm{~m}^{-2}$ at a gate voltage of $V_{g}=-50 \mathrm{mV}$ and $2.9 \times 10^{15} \mathrm{~m}^{-2}$ at $V_{g}=+100 \mathrm{mV}$, respectively.

At $V_{g} \neq 0$, we find a strong, gate voltage dependent positive magnetoresistance (PMR) around $B=0$ which can extend well above 0.7 T, where Shubnikov-de Haas oscillations set in. Commensurability resonances [1, 2, 3] are not observed. The Hall resistivity $\rho_{x y}$ is a straight line and depends only on the average electron density (not shown). This phenomenology is in tune with measurements on two-dimensional density modulations in square lattices, where the PMR is seen up to $\approx 200 \mathrm{mT}$ corresponding to the larger LSL periods, while commensurability resonances are observed in various strengths. [3, 16, 17] From semiclassical simulations, it has emerged

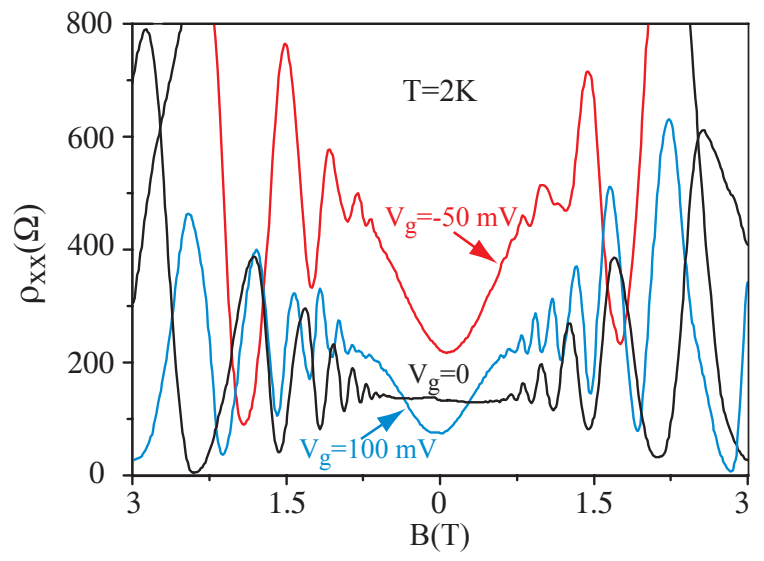

FIG. 2: (color online) Longitudinal magnetoresistance of the 2DEG underneath the gated region at different gate voltages.
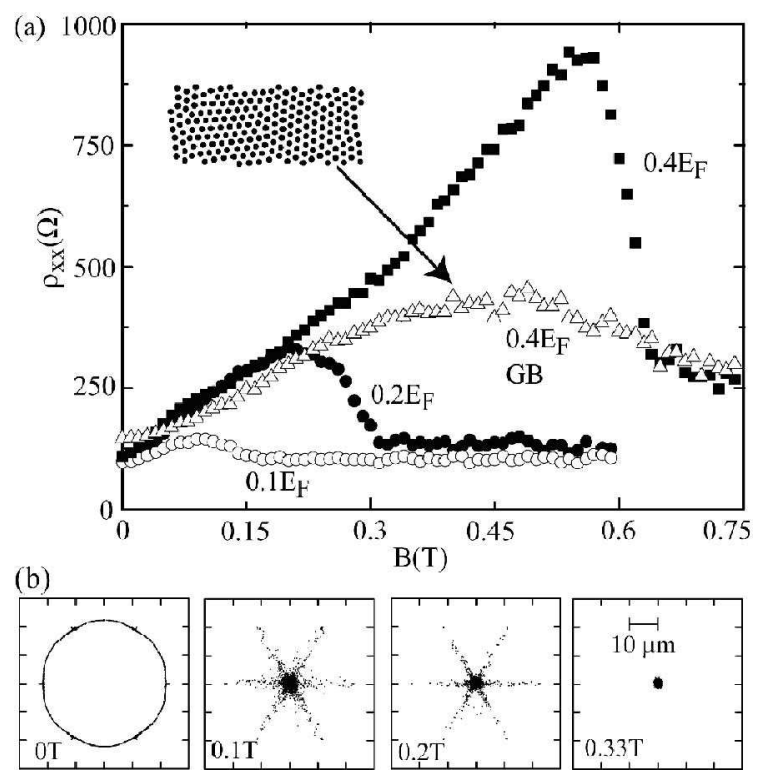

FIG. 3: (a): Simulated longitudinal resistivity for different modulation strengths $e V_{0}$ for a perfect lattice as well as for a LSL with grain boundaries for $e V_{0}=0.4 E_{F}$. (b): Final positions of 5000 electrons in different magnetic fields, after $10 \tau_{D}$ for $e V_{0}=0.2 E_{F}$.

that the PMR originates from $\vec{E} \times \vec{B}$ drifts guided along the corrugated potential channels of the LSL, and breaks down for cyclotron forces larger than the force exerted by the modulation potential, i.e., for $B \gtrsim V_{0} /\left(a v_{F}\right)$ where $V_{0}$ denotes the modulation potential amplitude and $v_{F}$ the Fermi velocity. 18] The strength of the commensurability resonances depends on the amplitude and symmetry of the modulation potential as well as on the disorder scattering. [18, 19, 20]

To substantiate this interpretation, we performed semiclassical simulations based on the Kubo formalism. 21, 22 The potential $V(x, y)=V_{0} \cos ^{2}(k d)$ with $d=\sqrt{\left(x-x_{c}\right)^{2}+\left(y-y_{c}\right)^{2}}$ and $k=\pi / a$ for $d<a / 2$ 
and $V=0$ for $d>a / 2$ was allocated to every point $\left(x_{c}, y_{c}\right)$ of the LSL. For each magnetic field, the semiclassical equations of motion were solved numerically for 40.000 electrons, and the conductivity tensor was calculated from the velocity correlation function via $\sigma_{i j}(B)=$ $\frac{m^{*} e^{2}}{\pi \hbar^{2}} \int_{0}^{\infty}\left\langle v_{i}(t, B) v_{j}(0)\right\rangle e^{-t / \tau_{D}} \mathrm{~d} t$, where $t_{D}=11 \mathrm{ps}$ and $v_{i}, i=x, y$ are the Drude scattering time and components of the Fermi velocity, respectively, while $\langle\ldots\rangle$ denotes emsemble averaging. The longitudinal resistivity $\rho_{x x}(B)$ is then obtained from inversion of the simulated magnetoconductivity tensor. For a perfect hexagonal LSL with $a=45 \mathrm{~nm}$, we find a pronounced PMR around $B=0$ which collapses at a magnetic field that increases as $V_{0}$ increases, see Fig. 3(a).

The evolution of the diffusion cloud in increasing magnetic fields provides insight in the origin of this structure, as exemplified for $V_{0}=0.2 E_{F}$ in Fig. 3. (b), where the end positions of 5000 electrons are plotted. They are injected with velocity $v_{F}$ from the area $[0, a] \times[0, \sqrt{3} a]$ in random directions after $110 \mathrm{ps}$ with disorder scattering turned off. At $B=0$, transport is essentially isotropic. As $B$ increases, the electrons are more and more guided along the six directions of high symmetry of the LSL, along which open orbits are possible. [18, 19, 20] Around $B=0.2 \mathrm{~T}$, the guiding collapses and $\rho_{x x}$ experiences a dramatic drop. Commensurability resonances are absent in the simulations as well. 18]

While these simulations nicely reproduce the measured PMR, the sharp drop of $\rho_{x x}$ is not seen experimentally. We attribute this deviation to the disorder in our LSL, as corroborated by magnetotransport simulations in disordered arrays with $V_{0}=0.4 E_{F}$. A hexagonal lattice containing grain boundaries was generated by a Monte Carlo method described elsewhere. 23] The corresponding simulated trace of $\rho_{x x}(B)$, see Fig. 3), shows a significant damping of the magnetoresistance peak, while the slope of the PMR close to $B=0$ remains basically unaffected.

In summary, we have generated a hexagonal LSL with a period of $43 \mathrm{~nm}$ in a $2 \mathrm{DEG}$ using diblock copolymer masks in the hexagonal cylindrical phase. A positive magnetoresistance around $B=0$ is found which extends into the Landau quantization regime. It has its origin in electron trajectories which are guided along the corrugated potential channels generated by the LSL. The disorder present in our DBC mask smears the magnetoresistance peak to be expected in perfect superlattices, but does not destroy the PMR. Since the Fermi wavelength is comparable to the superlattice period in our system, the validity of the semiclassical model is not clear, and it remains to be seen in further work how a quantum model compares to our measurements.

Financial support by the Heinrich-Heine-Universität Düsseldorf and the DFG (SFB 418) is gratefully acknowl- edged.

* Electronic address: thomas.heinzel@uni-duesseldorf.de

[1] K. Ensslin and P. M. Petroff, Phys. Rev. B 41, 12307 (1990).

[2] D. Weiss, M. L. Roukes, A. Menschig, P. Grambow, K. v. Klitzing, and G. Weimann, Phys. Rev. Lett. 66, 2790 (1991).

[3] A. Lorke, J. P. Kotthaus, and K. Ploog, Phys. Rev. B 44, 3447 (1991).

[4] F. Nihey, S. W. Hwang, and K. Nakamura, Phys. Rev. B 51, 4649 (1995).

[5] Y. Iye, M. Ueki, A. Endo, and S. Katsumoto, J. Phys. Soc. Japan 73, 3370 (2004).

[6] W. Kang, H. L. Stormer, L. N. Pfeiffer, K. W. Baldwin, and K. W. West, Phys. Rev. Lett. 71, 3850 (1993).

[7] M. C. Geisler, J. H. Smet, V. Umansky, K. von Klitzing, B. Naundorf, R. Ketzmerick, and H. Schweizer, Phys. Rev. Lett. 92, 256801 (2004).

[8] T. Schlösser, K. Ensslin, J. P. Kotthaus, and M. Holland, Europhys. Lett. 33, 683 (1996).

[9] C. Albrecht, J. H. Smet, K. v. Klitzing, D. Weiss, V. Umansky, and H. Schweizer, Phys. Rev. Lett. 86, 147 (2001).

[10] J. Pedersen, C. Flindt, N. A. Mortensen, and A.-P. Jauho, Phys. Rev. B 77, 045325 (2008).

[11] C. T. Black, R. Ruiz, G. Breyta, J. Y. Cheng, M. E. Colburn, K. W. Guarini, H.-C. Kim, and Y. Zhang, IBM J. Res. Dev. 51, 605 (2007).

[12] S. Melinte, M. Berciu, C. Zhou, E. Tutuc, S. J. Papadakis, C. Harrison, E. P. D. Poortere, M. Wu, P. M. Chaikin, M. Shayegan, et al., Phys. Rev. Lett. 92, 036802 (2004).

[13] The heterostructures have been purchased from Intelligent Epitaxy Tech., Richardson, TX (USA).

[14] T. Xu, A. V. Zvelindovsky, G. J. A. sevink, K. S. Lyakhova, H. Jinnai, and T. P. Russell, Macromolecules 38, 10788 (2005).

[15] T. Thurn-Albrecht, J. Schotter, G. Köstle, N. Emley, T. Shibauchi, L. Krusin-Elbaum, K. Guarini, C. T. Black, M. T. Tuominen, and T. P. Russell, Science 290, 2126 (2000).

[16] M. C. Geisler, S. Chowdhury, J. H. Smet, L. Höppel, V. Umansky, R. R. Gerhardts, and K. von Klitzing, Phys. Rev. B 72, 045320 (2005).

[17] S. Chowdhury, C. J. Emeleus, B. Milton, E. Skuras, A. R. Long, J. H. Davies, G. Pennelli, and C. R. Stanley, Phys. Rev. B 62, R4821 (2000).

[18] D. E. Grant, A. D. Long, and J. H. Davies, Phys. Rev. B 61, 13127 (2000).

[19] A. D. Mirlin, E. Tsitsishvili, and P. Wölfle, Phys. Rev. B 63, 245310 (2001).

[20] R. R. Gerhardts and S. D. M. Zwerschke, Phys. Rev. B 64, 115322 (2001).

[21] R. Kubo, J. Phys. Soc. Jpn. 12, 570 (1957).

[22] R. Fleischmann, T. Geisel, and R. Ketzmerick, Phys. Rev. Lett. 68, 1367 (1992).

[23] S. Klinkhammer, H. Xu, T. Heinzel, U. Gennser, G. Faini, C. Ulysse, and A. Cavanna, Phys. Rev. B 77, 235311 (2008). 